\title{
The US and Other Optically Selected Bright QSO Samples
}

\author{
Kenneth J. Mitchell \\ Science Applications International Corporation, 4600 Powder Mill Rd., \\ Suite 400, Beltsville, MD, 20705-2675 \\ Peter D. Usher \\ Dept. of Astronomy and Astrophysics, The Pennsylvania State Univ., \\ University Park, PA 16802
}

\begin{abstract}
The US Bright Quasar Sample (UBQS) is a color-selected sample of quasars with $B<17$ that has been constructed with an eye towards completeness within well-defined selection limits. The redshift distribution of the UBQS shows an interesting spike at $z \sim 0.55$. The significance of this enhancement increases when the UBQS is combined with other bright quasar samples which also show evidence of high levels of completeness. Reconstitution of this combined bright quasar sample after removal of the emission-line flux from the $B$ magnitudes indicates that the $z \sim 0.55$ spike is not caused by an emission-line selection bias. Rather, the largest effect of these sample corrections would be to depress the high end of the optical luminosity function that is derivable from this combined sample.
\end{abstract}

\section{Introduction}

Bright quasar samples make unique contributions to the study of the cosmological evolution of the quasar population by establishing the optical luminosity function at low redshifts and by determining the bright end of the optical luminosity function at all redshifts. However, historically the numbers of bright quasars in well-defined, complete samples have been relatively small because of both their intrinsically low surface density on the sky and the problems with selection effects in the necessarily large-scale bright-quasar surveys. Thus, the accuracy of the derived redshift-dependent quasar optical luminosity function at low redshifts and high luminosities has been uncertain.

The 19 member UBQS (Usher \& Mitchell 2000; herein UM2000) was established to help bolster the numbers of bright $(B<17)$ quasars in complete samples and to help further explore the properties of bright quasar surveys in general. The UBQS is derived from the US color-excess survey (Usher 1981; Usher \& Mitchell 1990) which features manual selection of candidates from 3color photographic plates. The US selection techniques have proven to be highly reliable and complete for the selection of unresolved objects with colors that are bluer (in $B-V$ ) and/or more ultraviolet (in $U-B$ ) than the metal-deficient halo subdwarfs (e.g. Mitchell, Warnock, \& Usher 1984; Mitchell 1998). Although the 
UBQS covers a modest $\sim 200$ square degrees, it provides a useful comparison with past bright-quasar survey work, and it can also be used to help test the properties of the modern, large-scale, digital optical/near-IR surveys reported on at this conference: the Sloan Digital Sky Survey (Ivezic 2002), the 2 Micron All Sky Survey (Cutri 2002), and the Hamburg/ESO Survey (HES: Wisotzki 2002) all of which show great promise for establishing large, complete samples of color-selected bright quasars.

This paper reviews the status of the UBQS and other pre-existing bright quasar samples. It also extends the results of UM2000 by exploring the effects on bright quasar samples when emission line flux is explicitly accounted for and removed from the quasar $B$ magnitudes.

\section{A Combined Bright Quasar Sample}

Since the numbers of quasars in existing individual bright samples are relatively small, it is necessary to combine samples to enhance statistical accuracy. The approach taken by UM2000 is to combine only those bright quasar samples (or subsamples thereof with $B<16.95 \mathrm{mag}$ ) which show signs of absolute completeness. Of the six bright quasar samples extant at the time, it was found that only three share the properties that: (a) their surface densities are close to the maximum detected values; and (b) their redshift distributions have shapes that are mutually consistent and that generally agree with the predictions of recent empirical models of the evolving quasar luminosity function (La Franca \& Cristiani 1997); these three are the UBQS, an early version of the HES (HES97: Köhler et al. 1997), and the Edinburgh Quasar Survey (EQS: Goldschmidt et al. 1992). The other three extant bright quasar samples were not chosen for combination by UM2000 for the following reasons: (1) the BQS (Schmidt and Green 1983) has low counts, and the $(U-B)$ color limit biases the redshift distribution; (2) the LBQS (Hewett, Foltz, \& Chaffee 1995) has low counts, a redshift distribution underpopulated at low redshift, and a claimed bright-magnitude limit that vacillates; (3) the HBQS (Cristiani et al. 1995) has very low counts. This relatively straightforward approach for the combination of quasar samples is complementary to those that strive to include more samples by attempting to correct for differing sample selection effects and/or errors; such effects can cause some quasar samples to deviate markedly in their overall properties from others. For example, the LBQS and HBQS were used in the La Franca \& Cristiani (1997) study of multiple quasar samples; however, the BQS was not - a curiosity, since La Franca \& Cristiani's methodology seems particularly well-suited to account for the well-defined selection effects of the BQS and since that sample potentially carries such a large weight at the brightest magnitudes.

The combined bright quasar sample constructed from the UBQS, EQS, and HES97 covers $\sim 1115$ square degrees and contains 53 quasars with $B<16.95$ mag and $0.2<z<2.2(0.3<z<2.2$ for the EQS $)$. These limits ensure selection completeness for quasars with starlike morphology on $1.2 \mathrm{~m}$ Schmidt imagery according to the morphology criterion established by Fabian \& Usher (1996). The surface densities of the combined sample closely match those predicted by the Luminosity Dependent Luminosity Evolution (LDLE) model of La Franca and Cristiani (1997). The shape of the observed redshift distribution (shown in 

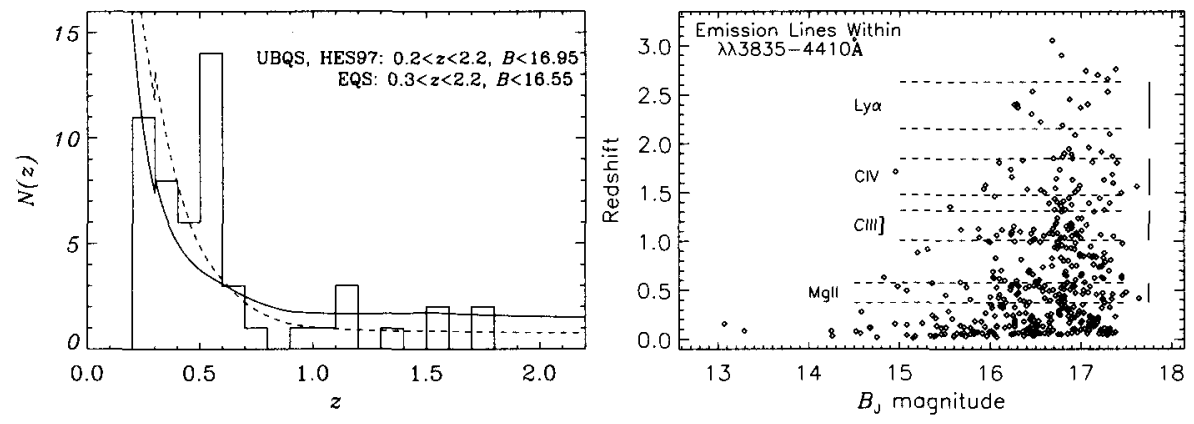

Figure 1. Left: Redshift distribution of the combined bright quasar sample compared to LDLE (dashed curve) and Pure Luminosity Evolution (PLE: continuous curve) model predictions. Right: Hubble daigram of the HESO0 sample. Redshift ranges are noted where the emission lines enter the $>85 \%$ response level of the $B_{\mathrm{J}}$ bandpass.

Figure 1, left side) also matches the general behavior of the LDLE model, except for the noticeable excess numbers in the $0.5<z \leq 0.6$ bin. The subject of this "spike" in the redshift distribution is taken up in the next section.

Since the UM2000 study, a new version of the HES sample has been published (HES00: Wisotzki et al. 2000). The size of this bright sample is impressive: 415 quasars with $B_{\mathrm{J}}<17.5$. However, as indicated in Figure 1 (right side), the HES00 Hubble diagram contains apparent horizontal striation. The unusual distribution of objects can be traced to a preference for the higher luminosity quasars to be located at redshifts where the major quasar emission lines enter the $>85 \%$ response level of the $B_{\mathrm{J}}$ bandpass. Unlike the $B$ magnitudes of the HES97 sample, the HES00 sample uses $B_{\mathrm{J}}$ magnitudes derived directly from the survey objective prism spectra. The suspect structure in the Hubble diagram might be an indication that the emission lines, via uncalibrated emulsion non-linearities or other effects, have biased the derived $B_{\mathrm{J}}$ magnitudes. For this reason the HES00 sample is not considered here; instead, the even larger HES quasar sample (Wisotzki 2002) is awaited. These considerations serve as a reminder of the potential effects that emission lines can have on quasar magnitudes and selection.

\section{The $z \sim 0.55$ "Spike": Real or Statistical Artifact?}

An intriguing feature of the combined-sample redshift distribution of Figure 1 (left side) is the obvious excess of quasars in the $0.5<z \leq 0.6$ bin. This excess consists of $\sim 10$ of the 14 quasars in that bin, as estimated both from the trends in the observed data and by comparisons with the model distributions. UM2000 assess the significance of the spike using the conservative Kolmogorov-Smirnov (KS) and Cramer-von Mises (CvM) statistical tests to compare the observed and modeled distributions. While the PLE model was rejected at the $99.9 \%$ level, LDLE could only be rejected at the $\sim 80 \%$ level, with the conclusion that the spike could just be a statistical artifact. The less conservative Chi-square test rejected both PLE and LDLE at the > 99\% level. 

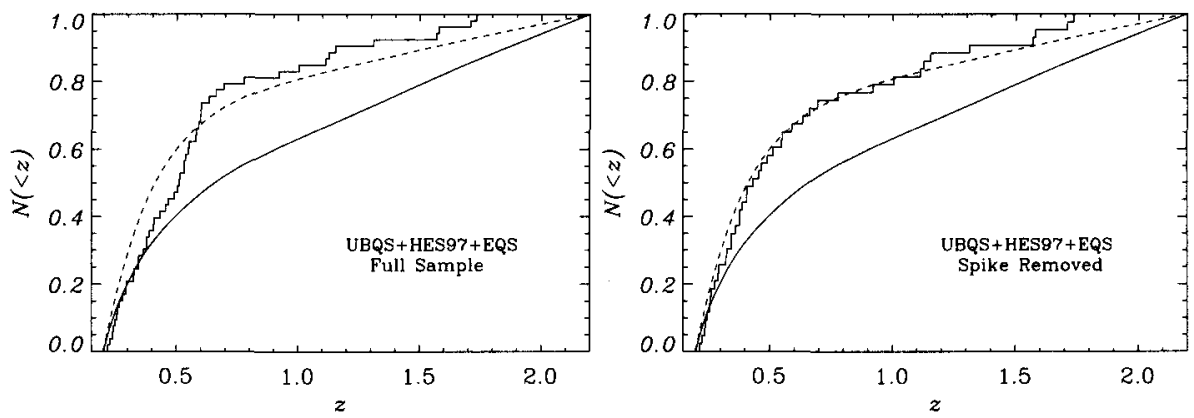

Figure 2. Left: Normalized, cumulative redshift distribution of the combined bright quasar sample compared to LDLE (dashed curve) and PLE (continuous curve) model predictions. Right: Same, except the excess within the $z \sim 0.55$ "spike" has been removed.

Another view of the data is provided in Figure 2 which shows the observed and modeled cumulative redshift distributions (normalized to unity) that are the basis for the KS and CvM tests. In the full-sample plot (on the left) the data are seen to be a better match, overall, to the LDLE model prediction than to the PLE model, in agreement with the statistical results. However the match is not entirely satisfactory due to the effects of the $z \sim 0.55$ spike. The plot on the right shows the results when the spike is removed: i.e. 10 quasars with $0.5<z \leq 0.6$ have been randomly removed. The agreement between the shape of the remaining redshift distribution and the LDLE prediction is seen to be excellent. But note that the removal of the 10 quasars reduces the combinedsample surface densities by $\sim 20 \%$ on average, so that the original agreement between the surface densities of the full combined sample and those of the LDLE model would no longer hold.

The evidence is not conclusive either for or against the reality of the $z \sim 0.55$ spike. The conservative statistical tests indicate that the agreement between the data (spike included) and the LDLE model is acceptable, if not entirely satisfying. However, if the addition of further complete bright quasar samples prove the spike to be real, then the quasar luminosity function at $z \sim 0.55$ is uniquely distorted, and it could be an indication of very large scale structure. Not only would the current LDLE and other evolutionary models need modifications to account for the spike, but they would also need to be modified to correctly account for both the quasar surface density and redshift distribution outside of the spike.

\section{Effects of Removing the Emission-line Flux from the Combined- Sample $B$ Magnitudes}

Emission-lines contribute flux to quasar magnitudes at certain redshifts and, unless accounted for, can lead to sample bias. Such a bias, caused by the MgII emission line entering the $B$ bandpass, could be one reason for the enhanced numbers of $z \sim 0.55$ quasars in the combined UBQS, HES97, and EQS sample. UM2000 made an initial estimate that most of the spike is not likely caused 

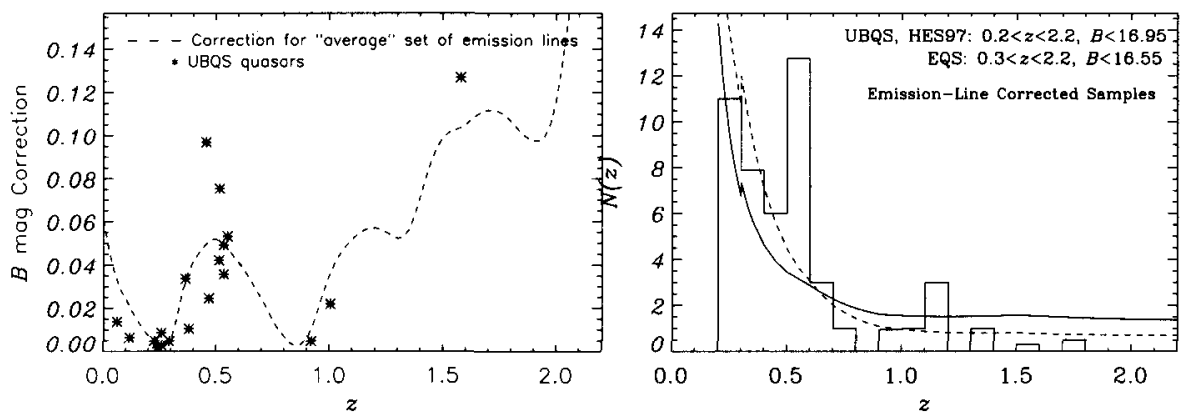

Figure 3. Left: $B$ magnitude corrections for the individual UBQS quasars due to removal of emission-line flux. The dotted line represents the correction for an assumed set of average emission-line EWs. Right: Redshift distribution of the "corrected" combined bright quasar sample after objects with $B_{\text {corr }}>B_{\lim }$ have been removed. Lines are as in Figure 1.

by the effects of the MgII line. This selection effect is more fully investigated here, given the potential consequences of detecting a real spike in the quasar redshift distribution. The method used corrects the $B$ magnitudes by removing any emission-line flux, and then removes from the combined sample any quasars with corrected magnitudes, $B_{\text {corr }}$, that are fainter than the faint magnitude limit $B_{\text {lim }}$ of their parent sample. This is a different approach than attempting to account for emission-line flux via the K-correction.

The magnitude correction due to the removal of emission line flux in the $B$ bandpass, $S_{B}$, is:

$$
\Delta B=2.5 \log \left[1+\sum_{1}\left\{\left(E W_{1} / \lambda_{1}^{\alpha}\right)\left(\int G_{1}(\lambda) S_{B}(\lambda) \mathrm{d} \lambda / \int \lambda^{-\alpha} S_{B}(\lambda) \mathrm{d} \lambda\right)\right\}\right]
$$

where $E W_{1}$ is the equivalent width of emission line $1, \lambda_{1}$ is the central wavelength of line $1, G_{1}$ is the Gaussian line profile of line 1 centered on $\lambda_{1}$, and $\alpha$ is the slope of the quasar continuum. The corrected magnitude is then $B_{\text {corr }}=B+\Delta B$.

All of these parameters have been measured or can be estimated for the UBQS, HES97, and EQS quasars in order to calculate $\Delta B$. For example, the $\triangle B$ calculated for the individual UBQS quasars based on measured EWs are shown in Figure 3 (left-hand side). Also shown in that same plot is the $\Delta B$ relation for a set of emission lines (Balmer, MgII, CIII], CIV, SiIV+OIV], Ly $\alpha$ ) with estimated "average" EWs. Measured EWs have not been published for the HES97 and EQS quasars and so a crude, Gaussian-like probability distribution centered on these average EWs is assumed for their EW strengths. Based on this EW probability distribution and the quasar's redshift, Monte-Carlo calculations then provide estimated probabilities of excluding each quasar from the sample based on the probability, $\mathrm{P}$, that $B_{\text {corr }}>B_{\lim }$. Most quasars have $\mathrm{P}=0$; quasars with non-zero $\mathrm{P}$ have been kept in the combined sample, but with a reduced weight $(=1-\mathrm{P})$.

The results indicate that a weighted total of $\sim 4.6$ quasars of the original 53 should be excluded from the corrected combined sample, an overall reduction 
of $\sim 10 \% .99 \%$ of this loss is attributed to the effects of 2 lines: MgII excludes 1.4 quasars, and CIV excludes 3.2 quasars. The resulting redshift distribution for the corrected, combined sample is shown in Figure 3 (right-hand side), and it can be compared with the original redshift distribution of Figure 1 (left-hand side). The $z \sim 0.55$ spike has lost only $\sim 14 \%$ of its members, and it remains prominent in the corrected sample. On the other hand 3.2 of the original 4 high-redshift $(z>1.5)$ quasars have been excluded from the corrected combined sample, a reduction of $80 \%$ ! KS and CvM statistical tests indicate that the match with LDLE remains statistically acceptable, albeit dubious.

It is concluded that the $z \sim 0.55$ spike in the bright quasar redshift distribution is very likely not caused by emission-line bias in the $B$ magnitudes, and thus it remains a candidate as an unusual feature in the quasar luminosity function. The largest effects of correcting the $B$ magnitudes has been at high redshifts, where the resulting sample corrections depress the bright end of the optical luminosity function that is derivable from the combined sample. This is in essential agreement with one of the cautionary notes struck by Wampler \& Ponz (1985).

\section{References}

Cristiani, S., et al. 1995, A\&AS, 112, 347

Cutri, R. M. 2002, these proceedings

Fabian, D., \& Usher, P.D. 1996, AJ, 111, 645

Goldschmidt, P., Miller, L., La Franca, F., \& Cristiani, S. 1992, MNRAS, 256, $65 \mathrm{P}$

Hewett, P.C., Foltz, C.B., \& Chaffee, F.C. 1995, AJ, 109, 1498

Ivezic, Z. 2002, these proceedings

Köhler, T., Groote, D., Reimers, D., \& Wisotzki, L. 1997, A\&A, 325, 502

La Franca, F., \& Cristiani, S. 1997, AJ, 113, 1517 Err.: 1998, AJ, 115, 1688

Mitchell, K.J. 1998, ApJ, 494, 256

Mitchell, K.J., Warnock, A. III, \& Usher, P.D. 1984, ApJ, 287, L3

Schmidt, M. \& Green, R.F. 1983, ApJ, 269, 352

Usher, P.D. 1981, ApJS, 46, 117

Usher, P.D., \& Mitchell, K.J. 1990, ApJS, 74, 885

Usher, P.D., \& Mitchell, K.J. 2000, AJ, 120, 1683

Wampler, E.J. \& Ponz, D. 1985, ApJ, 298, 448

Wisotzki, L. et al. 2000, A\&A, 358, 77

Wisotzki, L. 2002, these proceedings 